\title{
The hope of personalized medicine
}

\author{
Peter Kapitein* \\ CEO \& Patient Advocate, Inspire2Live, The Netherlands
}

\begin{abstract}
Personalized Medicine is a very popular topic in the literature of oncology nowadays. One can understand different things of this subject but there is a kind of common sense that at least we know that in each patient the tumor has it's own characteristics and needs a treatment that is tailored along these characteristics. It's strange that this is already known where so many patients are still treated along the line of the 'old protocols'. With modern diagnostics like DNA sequencing and testing drugs on small slices of tumor tissue it is possible to fine-tune the treatment. It is also possible to re-biopsy in a very patient friendly way with liquid biopsies so that we can 'follow' the tumor as it changes during treatment or maybe because of this treatment. A changing tumor needs a changing treatment. This new way of diagnosis and $n=1$ tailor made treatment needs changes in the way we think about cancer as a disease in a particular organ, it needs changing of the work of regulators that register drugs for organs instead of registering it for a certain kind of DNA-mutation and the way industry works will also needs to be worked out in a different way. All this is possible although very hard to do. The urge to do so however is found in the fact that we now know how to prevent the loss of many lives where in the past we did not. Hope is our driving force.
\end{abstract}

\section{Opinion}

"Inaction kills". It was Joep Lange [1] who brought this up regularly. If you do not act, this leads to victims in health care. That is why we must act and do this especially on the basis of facts, truthful arguments and intentions that are pure. And not only in the rich, white, western world.

\section{Personalized medicine and diagnostics}

More and more I am involved in discussions around "Personalized Medicine". The expression "Precision Medicine" also exists. It seems that each year a different and even more beautiful expression for the same idea appears. Anyway, this makes clear that we are convinced that cancer is an individual disease and has to be geared to the individual. That cancer in one body differs from cancer in another body and thus requires a very carefully tuned treatment. This assumption has existed for a long time and actually we have known it for some time, but really proving it in a thorough and scientific way has not often been done yet. However, the first successes in this field begin to be visible now. An important and controversial article by René Bernards showed this from 2010 among other things [2]. Better and better we are able to determine what is going on in an individual cancer patient tumor-technically. Diagnosis plays a decisive role in this.

Moreover, in the meantime we know that cancer does not remain unchanged. Why this is so may not be completely clear. Does the cancer mutate by itself or because we besiege it with actions? Or might it be a combination of these two? Or yet a different and unknown reason? Anyway, it is obvious that in many cases the cancer won't give up after one hit on its head. We play chess with cancer and that is the reason why we not only have to apply other tactics (other treatments), but also have to think ahead. Only the grandmaster wins.

\section{The old protocols}

For a number of years we have had this knowledge and as a matter of fact this knowledge increases every year, but still in many cases we treat cancer along protocols that use the common denominator: a woman has "ovary cancer", a man has "prostate cancer", and someone has "colon cancer". We treat along the lines of the organ and have a treatment for that. Some time ago we looked it up and thought of it. We have standard treatments for it and in many cases we still apply these. In the meantime we know better. At this moment we give treatments to patients of whom we know, or at least may know, that they do nothing else than making patients sicker than they already are. And what may even be worse is that we still start and carry out trials that do nothing but damage patients. During the Annual Congress of Inspire2Live in 2015 professor and physician Sabine Tejpar showed in an upsetting way that (at that moment, January 2015) there is no trial in the field of colon cancer that is advantageous for the patient [3]. Our ability to turn new scientific knowledge into clinical practice fails badly.

\section{Innovation of the clinical practice}

There is a discrepancy between science and clinical practice. At least in most cases, for in academic centers science is transferred to clinical practice faster, although here speed is not very high either. However, this has nothing to do with reluctance, but with the way the trials are set up and the implementation of a successful trial into practice. I will come back to this later when I write about the required changes in the research into and the registration of new medicines and the changes in the care around cancer patients.

\section{There is more than only medicines}

When designing and developing new treatments we often think of medicines right away, whereas we know that patients that have really been cured owe this to surgery and radiotherapy. Of course these two

Correspondence to: Dr. h.c. Peter Kapitein, CEO \& Patient Advocate, Inspire2Live, Noorderakerweg 271B 1069 LT Amsterdam, The Netherlands, E-mail: peter.kapitein@gmail.com

Received: May 01, 2016; Accepted: May 19, 2016; Published: May 23, 2016 
forms have excellent results with tumors that have not metastasized. With tumors that have formed metastases it is more difficult to bring about cures with these techniques, but also here there is hope. I seriously think that in the next few years the intervention oncology will develop as a new surgical branch: Surgery without incision. Not as a replacement, but as an essential added form of surgery. This technique can also be used for the treatment of metastases very well. Again, not as a cure yet, but as a form of surgery that is very patient friendly and just as effective in fighting metastases. I am thinking about the treatment of metastases to the liver of colon cancer and the treatment with Radio Embolization [4]. In the Netherlands this form of treatment has been added to the basic care package lately. I think that applying this form of "operating" can also be described as "Personalized Medicine". What is nice and good of Intervention Oncology is that it is a hopeful combination of "surgery" (without incision), radiology and treatment with medicines. With very beautiful, good and modern techniques we are able to administer medicines extremely locally. With the desired effect on the tumor and many undesirable side effects staying away. Let us please keep thinking broadly with "Personalized Medicine" and not only overload a patient with medicines in a way the old protocols prescribe.

\section{Hope as driving force}

Medicines work pretty well with blood cancers, but with solid tumors there is still incremental tinkering. Immunotherapy, however, is hopeful and that is what we have to focus on. Hope, however, is the word that gets me to act. "Hope" is a driving force and it causes action. If "inaction kills", we need hope to act. And that hope comes from "Personalized Medicine". That is my sincere conviction. But then we have to implement it. Fully and fast. We are better and better able to map what is exactly going on in a patient's body in front of a doctor. Diagnostic techniques are essentially different and better than ten years ago. But they are only used in a restricted number of hospitals and for a limited number of patients, mostly in trials. If you are no patient in such a hospital and are not in a trial, you are treated as a "normal colon cancer patient" and you have to deal with the protocol. You are supposed to belong to the common denominator group and if that is the case, you are lucky and are cured. If it is not, you die.

\section{Diagnostics in a new dress}

We now understand that each tumor is different at the molecular level. On the basis of the mutation in the tumor we can decide what is going on in your tumor (what change decides that you get ill). Lately this can also been done by sequencing the DNA of the tumor. Then it is established what medicine attacks your individual tumor characteristic. With new diagnostic techniques it is possible to fine-tune the correct treatment to the individual patient. With these techniques we must not only think of sequencing the tumor. That has to be done anyway and has to be implemented as standard procedure for all patients. Patients must no longer accept a treatment that does not use the conclusions of determining the DNA mutation. After all, we know that we treat many patients unnecessarily in that case. Recently it was shown that the MamaPrint (that exists for ten years now) can prevent many women (only in the Netherlands 2.500 women per year) from getting chemotherapy that does nothing else than making them more sick and that has serious side effects. Without consequences for the tumor this chemotherapy can be left out [5]. And yes, something like this can be costly, but it is more important that we no longer burden patients with nasty side effects.
Apart from this, in the ErasmusMC they also work with detecting DNA mutations by means of tests of very thin slices of tumor tissue, which can be kept alive in a laboratory and to which a new or existing therapy can be applied first to decide if the treatment has the desired effect [6]. In this way, for example, a test has been developed that determines if a breast cancer tumor has a defect in a DNA repair process, called Homologous Recombination, through which that tumor is extremely sensitive to a new medicine (Olaparib, brand name Lynparza), which uses this flaw of the tumor [7]. In this way the correct medicine can be given to the right patient. This is very successful as well and it contributes to a qualitatively much more effective treatment with far fewer side effects.

We see that in the last few years diagnostics have changed enormously and that the quality of the diagnosis has been improved strongly. Less and less our problem is making the right diagnosis. Our problem is organizing it in such a way that all patients get this new and better diagnosis and the resulting better treatment with often fewer side effects. The problem is that scientists and doctors have not been educated to organize. And they must not be taught. They have to do research and cure patients. Organizing is a profession and others can do that. It is all about the ideal cooperation between the organizers in health care and the patients, doctors and scientists. It is both plain and easy to solve. And money is not the problem. There is sufficient money in health care. It is all about the way we apply it. It is all about our will.

\section{The tailor made treatment}

A few years ago an excellent initiative was started in the Netherlands: the Center for Personalized Cancer Treatment (CPCT) [8]. What has not been organized well so far is that CPCT, in line with national guidelines, only treats patients when this guideline has been followed first. In other words: first the treatment belonging to the guideline has to be followed. Even if research and diagnosis at CPCT could make clear that this treatment is not suitable for a patient, because the data simply shows that the medicine won't attack the mutation in this individual patient's body (think for example of the research just mentioned, which makes clear that chemotherapy for breast cancer patients is unnecessary in many cases). We know that the chances for the patient diminish if he/she does not get the right treatment immediately, not to speak of the extra stress. Also here we see that clinical practice is not based on scientific knowledge and experience. Patients have to wait and accept that they get a worse or even useless and sickening treatment before they are allowed a treatment by CPCT according to the principles of "Personalized Medicine". There is work to do here and preferably as soon as possible. Work that has to be started not only by the patients, but also by doctors and scientists towards regulators and health care insurance companies.

\section{Re-biopsy for a better fine-tuning between treatment and patient}

What does not often happen is taking a further biopsy after the treatment. This is necessary because for various reasons and through time a tumor may change. In "targeted therapy" it sometimes occurs that a tumor chooses a detour or a different pathway. In that case treatment has to be adapted again. That happened to me already in 2005. After three standard therapy chemotherapies did not appear to work, my doctor and I were told by CT scan and bone marrow puncture.

Very strange is the argument that I heard recently: that a rebiopsy would not be ethical. Strange, because, as I said, I had many re- 
biopsies. I thought this self-evident. And yes, a bone marrow puncture is burdening and stressful, but you are happy to accept this. It is getting stranger yet, because patients have not been asked if this is ethical or not. Bureaucrats draw this conclusion in the absence of patients. This is not only strange or stranger, it is simply wrong. A patient thinks a re-biopsy stressful and self-evident at the same time.

Fortunately, there is more hope for the patient. Since recently re-biopsy does not have to be so burdening any longer. With socalled "liquid biopsies" it is possible to diagnose very precisely on the basis of blood. Since the tumor releases DNA to the bloodstream it is now possible to enrich this and make it suitable for sequencing [9]. Reliability of testing gene-mutation is $98 \%$ and it works in about $70 \%$ of all cases. With this reliability it is even better possible to tune in treatment to the individual. The patient in front of you. This new form of diagnosis works well after it has been determined that there is a tumor on the basis of histological research. It cannot be applied yet for screening healthy people. This will surely come, but cannot be done yet.

\section{$\mathrm{N}=1$. How is it done?}

$\mathrm{N}=1$ is not only necessary, it is also possible now and if it is implemented well, quality will be considerably improved in medical sense, perception of the quality of life by the patient and his/her loved ones will be great and the costs will also decrease. I am convinced of this last aspect, because I often see that the best quality leads to long term saving of costs. Giving fewer medicines will especially save money. As already said, we know that medicines do not often work and do nothing but making patients sicker and weaker than they already are. By stopping this and by only giving medicines of which we can say with much probability that they will work well, we use fewer medicines and save money. Besides, we need to spend far less money on treating side effects of existing ineffective treatments.

This new $\mathrm{n}=1$ form of treatment on the basis of better diagnosis has to be filled in practically as soon as possible to realize the advantages for patients as fast as we can. To be able to do this the necessary changes will have to be implemented. In any case they are changes

1) With regard to thinking about organs, as can be seen with doctors, also those that are educated now, and which have been scientifically outdated insights in the meantime

2) Of existing practice of regulators

3) For market orientation. The pharmaceutical industry will also have to change considerably.

\section{Ad 1) thinking about organs}

From the moment cancer starts people think about it in terms of cancer of an organ. And the whole chain of suspicion of cancer, referring a patient by the general practitioner, diagnosing the tumor (or determining that it is no cancer, which is the case 9 out of 10 times), treating the patient and her aftercare starts from here. Apart from some good initiatives this is how it works. For a part Personalized Medicine turns this upside down. We look at the DNA mutation, which may occur in several organs. Thus BRAF may be found in a melanoma, but also for instance in colon cancer. A better example, however, is ALK. ALK can be inhibited and in most cases the fusion partner (there are many possibilities) is not relevant. Examples are EML4-ALK in the lungs, CARS-ALK in myofibroblastic sarcoma. A medicine attacks this mutation, but in some cases that medicine cannot be administered, because it has been registered for one organ, but not for the other.
In fact, it can be given, but it is not, because hospitals do not get it reimbursed. For patients this is a tragedy, for their tumor might react positively on the medicine that is not registered for this tumor. Patients are missed out and will die without an assessment of its effects. They have not had a chance at all. The rules have been followed precisely, nobody can be reproached, but morally it is not correct. It will be clear that these problems will only become bigger with combination therapies (of possibly different manufacturers).

Everyone understands that this way of working must not be held up. In forced attempts to do something about this variations of existing themes are created. Then we get good initiatives like DRUP (Drug Rediscovery Protocol), PRIME (Primary Medicine) and SPECTA (Screening Patients for Efficient Clinical Trial Access). They are absolutely well meant and have some advantages, but they do not attack the problem at its core. They leave the existing system of thinking about organs and the attuned protocol for the design, layout, patient inclusion, evaluation and organization of trials intact. But Personalized Medicine contradicts to thinking about organs. It starts with the individual patient with a DNA mutation.

\section{Ad 2) the regulator}

The regulator registers in matters of "the state of science and practice". There must be evidence that a medicine is effective. Practically this often means that it works better than a placebo or an existing medicine, for a particular disorder. Besides, scientific literature must have been published about it that shows "evidence based" that it works. Of course regulators also struggle with Personalized Medicine and just as with "thinking about organs", described earlier, variations to existing themes are created here as well. The system of registration and the compensation, often attached to it, are creaking everywhere. The moment is there that also this part of cancer care returns to the designer's desk and that there will be decisions about how registration under Personalized Medicine will take place. A very good option will be to register a medicine for the DNA mutation after phases 1 and 2 and when applied on the basis of new and modern diagnostic techniques continue registering carefully how patients undergo treatment. Do not be afraid that useless treatments will be given. No patient wants a treatment that does not work. When these treatments are given there are other motives than those of the patient. Registration of the progress of the treatment leads to many new data, which can be applied again to optimize the system further. We create a learning system.

\section{Ad 3) market orientation}

Of course the way in which medicines are tested cannot be held up when $n=1$. For phase 1 we already need a dozen patients. For phase 3 this number will be considerably larger. This is not in proportion with $n=1$. A huge reconsideration will be necessary to test medicines and market them. It is not sensible to experiment with new medicines unfocused, but it is clear that they have to be brought to the patient faster than at the moment.

As described, I am aware of initiatives like DRUP, PRIME and SPECTA (and there will be some more), but they remain based on thinking as took place in the last few decades and they are not tuned to the new manner of diagnosing and creating a treatment plan in which the patient is considered a unique individual with his/her unique form of cancer, which has to undergo biopsies time and again. Variations to an existing theme, when designing, setting up and organizing trials, when treatment has changed rigorously, is asking for trouble. This won't succeed and in that case Personalized Medicine will die in beauty. 
A good and alternative approach is that after phase 2 the medicine is given to all patients with the gene mutation that is attacked by the medicine and of which the doctor, together with the patient, decides that it is sensible to administer the medicine. In that case the manufacturer makes the medicine available immediately and without cost for all patients for whom the doctor prescribes it. After three months (or sooner if there is a medical necessity) it is determined for whom it works and for these patients treatment is continued and paid for. With other patients treatment is stopped and if necessary a different treatment is selected. It is a matter of course that a manufacturer is given no financial compensation for a treatment that is not effective. Official registration takes place on the basis of the findings in this path.

\section{Conclusion and recommendation}

It should be possible to register and prescribe a medicine for a particular DNA mutation after phases 1 and 2. After this registration it is important to monitor the treatment carefully and to register what is happening in the patient. After that new biopsies have to be taken continually to get an optimal treatment. Treatment can be adapted where possible and necessary. A good registration will eventually improve the quality of the treatment, because we are setting up a learning system. A hundred times $\mathrm{N}=1$ makes $\mathrm{N}=100$. Measuring is knowing and with more knowledge Personalized Medicine can be made even more personalized.

\section{Hope becomes reality}

For all this a huge change in the method of working of health care is necessary, an industry not known for its inclination to change. The
German researcher Gerd Gigerenzer once compared it to moving a cemetery: "Changing healthcare is like moving a cemetery". But I know for sure that it is possible. If it is desirable for all stakeholders, is a different matter. However, "Inaction kills" and patients must not die unnecessarily.

\section{References}

1. https://en.wikipedia.org/wiki/Joep_Lange

2. Bernards R (2010) It's diagnostics stupid. Cell 141: 13-17. [Crossref]

3. Sabine Tejpar at the Amsterdam Inspire2Live Annual Congres 2015 about “Accelerate bench to bedside, how to learn faster':

4. http://mediasite.mediamission.nl/Mediasite/Play/efea922a50e849a5aa82be03714c3fd b1d?catalog=8eefdd31-492f-48f5-829b-f241784eae73

5. Vente MA1, Wondergem M, van der Tweel I, van den Bosch MA, Zonnenberg BA et. al. (2009) Yttrium-90 microsphere radio embolization for the treatment of liver malignancies: a structured meta-analysis. Eur Radiol 19: 951-959. [Crossref]

6. Website Antoni van Leeuwenhoek Ziekenhuis: Chemotherapie niet altijd nodig http://www.avl.nl/topmenu/over-avl/nieuws/groot-europees-onderzoek-laat-zienaanvullende-chemotherapie-bij-borstkanker-niet-altijd-nodig/

7. Naipal KA, Verkaik NS, Sánchez H, van Deurzen CH, den Bakker MA, et al. (2016) Tumor slice culture system to assess drug response of primary breast cancer. $B M C$ Cancer 16: 78. [Crossref]

8. Naipal KA, Verkaik NS, Ameziane N, van Deurzen CH, Ter Brugge P, et. al. Functional Ex Vivo Assay to Select Homologous Recombination-Deficient Breast Tumors for PARP Inhibitor Treatment. Clin Cancer Res 20: 4816-4826. [Crossref]

9. http://www.cpct.nl/nl/home/

10. Gautschi O, Aebi S, Heukamp LC (2015) Successful AZD9291 Therapy Based on Circulating T790M. J Thorac Oncol 10: e122-123. [Crossref]

Copyright: (C2016 Kapitein P. This is an open-access article distributed under the terms of the Creative Commons Attribution License, which permits unrestricted use, distribution, and reproduction in any medium, provided the original author and source are credited. 